Politique et Sociétés

\title{
Constitutionnalisation de la liberté d'association au Canada et négociation des rapports collectifs de travail dans les secteurs public et parapublic au Québec
}

\section{Yvan Perrier}

Volume 29, numéro 2, 2010

URI : https://id.erudit.org/iderudit/045170ar

DOI : https://doi.org/10.7202/045170ar

Aller au sommaire du numéro

Éditeur(s)

Société québécoise de science politique

ISSN

1203-9438 (imprimé)

1703-8480 (numérique)

Découvrir la revue

Citer cette note

Perrier, Y. (2010). Constitutionnalisation de la liberté d'association au Canada et négociation des rapports collectifs de travail dans les secteurs public et parapublic au Québec. Politique et Sociétés, 29(2), 131-153.

https://doi.org/10.7202/045170ar
Résumé de l'article

Dans le texte qui suit, il sera question de la notion de liberté d'association en droit canadien. De manière plus spécifique, nous examinons ce que la Charte canadienne des droits et libertés prévoit en matière de liberté d'association. Notre étude vise à analyser d'un peu plus près comment les acteurs sociaux que sont les juges de la Cour suprême du Canada ont interprété la notion de liberté d'association durant la période allant de l'adoption de la Charte canadienne des droits et libertés (en 1982) jusqu'à la décision de la Cour suprême du Canada dans l'arrêt Health Services and Support (en 2007). Peut-on s'attendre, à la suite de cet arrêt de 2007, à la fin de « l'exceptionnalisme permanent » (selon l'expression de Panitch et Schwartz, 1988) en matière de négociation des rapports collectifs de travail dans les secteurs public et parapublic au Québec? 


\title{
Note de recherche
}

\section{Constitutionnalisation de la liberté d'association au Canada et négociation des rapports collectifs de travail dans les secteurs public et parapublic au Québec}

\author{
Yvan Perrier \\ Cégep du Vieux Montréal \\ yperrier@cvm.qc.ca
}

« [O]n ne peut "jouir" des droits qu'en les exerçant.»

(Habermas, 1992: 446)

\begin{abstract}
Dans le texte qui suit, il sera question de la notion de liberté d'association en droit canadien. De manière plus spécifique, nous examinons ce que la Charte canadienne des droits et libertés prévoit en matière de liberté d'association. Notre étude vise à analyser d'un peu plus près comment les acteurs sociaux que sont les juges de la Cour suprême du Canada ont interprété la notion de liberté d'association durant la période allant de l'adoption de la Charte canadienne des droits et libertés (en 1982) jusqu'à la décision de la Cour suprême du Canada dans l'arrêt Health Services and Support (en 2007). Peut-on s'attendre, à la suite de cet arrêt de 2007, à la fin de "l'exceptionnalisme permanent» (selon l'expression de Panitch et Schwartz, 1988) en matière de négociation des rapports collectifs de travail dans les secteurs public et parapublic au Québec?

This article discusses the notion of freedom of association in Canadian law. More specifically, it examines what the Canadian Charter of Rights and Freedoms states concerning freedom of association. Our study takes a closer look at how the judges of the Supreme Court of Canada interpreted the notion of freedom association during the period from the adoption of the Canadian Charter of Rights and Freedoms (in 1982) until the Supreme Court of Canada's Health Services and Support decision (in 2007). May we expect, following this ruling, the end of "permanent exceptionalism » (according to the expression of Panitch and Swartz, 1988) regarding the negotiation of collective agreements in the public and parapublic sectors in Quebec?
\end{abstract}




\section{Introduction}

Tout au long des dix-neuvième et vingtième siècles, le mouvement ouvrier organisé a mené de nombreuses luttes économiques et politiques en vue de permettre une amélioration des conditions de travail, de rémunération, de vie et d'existence ainsi qu'un élargissement des droits démocratiques des membres de la classe ouvrière (CEQ/CSN, 1984; Rouillard, 2004). Parallèlement à ces luttes qui, à l'occasion, se sont accompagnées de grandes manifestations, les représentants des organisations syndicales se sont également, à certaines reprises, adressés aux tribunaux en vue de tester la portée constitutionnelle de la liberté d'association. C'est surtout depuis l'entrée en vigueur de La Charte des droits et libertés (Charte), en 1982, que les organisations syndicales ont commencé à exercer leurs droits de recours devant les tribunaux en vue de faire reconnaître la portée et l'étendue de la liberté d'association telle que définie à l'alinéa 2 d) de la Charte. Ainsi,

[a]vant l'adoption de la Charte canadienne des droits et libertés, la liberté d'association était un concept pour ainsi dire inconnu sur la scène juridique canadienne. Bien qu'inscrite au nombre des libertés et des droits humains fondamentaux de la Charte canadienne (comme complément de la liberté de réunion, toutefois) et, au Québec, de la Charte des droits et libertés de la personne, la liberté d'association avait rarement été invoquée dans la pratique. Dans chaque cas, d'ailleurs, on avait jugé qu'il n'y avait pas eu atteinte à la liberté1. (Bendel, 1986:322)

À ce sujet, dans le Renvoi relatif à la Public Service Employee Relations Act (Alberta), la Cour suprême du Canada (CSC) a refusé d'accorder une protection constitutionnelle à la poursuite des activités pour lesquelles l'association a été formée (RCS [Recueil de la Cour suprême du Canada], 1987: 313). En juin 2007, dans l'arrêt Health Services and Support, la Cour suprême du Canada écarte la position prise vingt ans plus tôt et conclut que la liberté d'association, garantie à l'alinéa 2 d) de la Charte, comprend également le droit pour les salariés et les salariées syndiqués de négocier collectivement leurs conditions de travail (CSC, 2007: 87, 90 et 92).

Dans le texte qui suit, il sera question de la notion de liberté d'association en droit canadien. De manière plus spécifique, nous examinerons ce que la Charte prévoit en matière de liberté d'association. Nous indiquerons également dans quelles perspectives cette liberté d'association a été introduite par les rédacteurs de la Charte. Nous analyserons ensuite l'apport de la Cour suprême, devenue l'arbitre judiciaire des droits et libertés et de leurs limites (partie 1). Nous entendons, à travers certaines décisions importantes de la Cour suprême, retracer ce mouvement qui va d'une vision majoritaire fort restrictive de la liberté d'association à son affirmation majoritaire positive comme incluant aussi une des finalités des regroupements de salariés: le droit de négociation. Nous regarderons comment les juges de la Cour suprême du Canada ont pris en considération la jurisprudence issue du droit international quand ils ont eu à interpréter la portée de la liberté d'association en matière syndicale (partie 2). Nous chercherons également à dégager l'impact de l'arrêt Health Services and Support (en 2007) sur les négociations des conventions collectives de travail dans les secteurs public et parapublic et à cerner les nouvelles

1. Au sujet de l'accueil des contestations juridiques de la part des syndicats devant les tribunaux, le professeur Harry W. Arthurs (2007:58) note: «The structure and powers of the judiciary are potentially of great importance in determining the scope and efficacy of labour law. Until quite recently, Canadian courts were generally unsympathetic, and often actively hostile, to workers and unions. » 
interactions possibles entre les acteurs concernés par ces négociations (le gouvernement du Québec et les organisations syndicales). Peut-on s'attendre, à la suite de cet arrêt, à la fin de «l'exceptionnalisme permanent» (selon l'expression de Panitch et Schwartz, 1988:15) en matière de négociation des rapports collectifs de travail dans les secteurs public et parapublic (partie 3)? Enfin, au moment où il est question d'une révision du régime de négociation dans les secteurs public et parapublic (Lévesque, 2007: A3; Richer, 2008: b1), quel avenir peut-on envisager pour la négociation dans ces deux secteurs? La nouvelle jurisprudence peut-elle avoir pour effet d'empêcher la mise en place d'un régime de négociation «de façade » (CSC, 2007: 104) (partie 4)?

\section{La notion de liberté d'association en droit canadien}

Depuis 1982, l'alinéa 2 d) de la Charte garantit l'existence de la liberté d'association. Cette garantie est assortie de deux limites. L'article 1 de la Charte prévoit que cette liberté peut être restreinte «par une règle de droit, dans des limites qui soient raisonnables et dont la justification puisse se démontrer dans le cadre d'une société libre et démocratique». L'article 33 autorise le Parlement ou les assemblées législatives à déroger à certaines des libertés garanties par la Constitution pour une période de cinq ans (comme le précise le paragraphe 4 de l'article 33, cette dérogation peut être renouvelable). Les lois adoptées par les deux paliers de gouvernement doivent, sous réserve des limites prévues aux articles 1 et 33, respecter les droits et libertés garantis dans la Charte (CSC, 2007: 104). Dans le Renvoi relatif à la sécession du Québec (RCS, 1998: 217), les juges de la Cour suprême ont identifié quatre principes fondamentaux (principes non écrits) à la base du droit constitutionnel canadien: le fédéralisme, la démocratie, le constitutionnalisme et la primauté du droit, et le respect des minorités. Le principe de la primauté du droit revêt une importance décisive puisqu'il figure dans le préambule de la Charte: «Attendu que le Canada est fondé sur des principes qui reconnaissent la suprématie de Dieu et la primauté du droit». Pour les juges de la Cour suprême du Canada, le principe de la primauté du droit s'impose de manière prééminente, il «exige que les actes du gouvernement soient conformes au droit, dont la Constitution» (cité par Coutu et Marceau, 2007: 77). Il appartient dès lors aux tribunaux de vérifier si la loi (ou certaines de ses dispositions) est conforme aux libertés et aux droits accordés aux individus par la Charte. L'adoption de la Charte n'a pas été sans effet sur le rôle des tribunaux dans la vie politique au Canada.

\section{Sur la redéfinition des relations entre le pouvoir législatif, exécutif et judiciaire au Canada depuis 1982}

La Charte a eu pour effet de modifier en profondeur le droit canadien (Bastarache, 2003:373). Nous pouvons même dire que nous sommes passés «du système de la suprématie parlementaire à celui de la suprématie constitutionnelle» (RCS, 1998: 493, 563; cité par Brunelle et al., 2007: 21). Depuis l'adoption de la Charte, en 1982, les tribunaux se sont vus investis de la responsabilité de scruter les lois pour assurer leur conformité aux valeurs fondamentales protégées par la Charte. Leur rôle de «gardiens de la Constitution", assumé depuis la fondation même du pays, s'enrichissait d'un chapitre nouveau important. Il appartient aux juges de la Cour 
suprême du Canada d'agir en tant que grands interprètes de la loi fondamentale du pays. Ils ont à décider de l'application des principes que l'on retrouve dans la Charte. La part prise par les tribunaux dans le processus politique n'est donc pas négligeable. Le pouvoir judiciaire est de plus en plus en mesure de freiner le pouvoir politique (Tremblay, 2005: 411-438). Depuis 1982, le Parlement canadien et les assemblées législatives doivent partager leur domaine d'intervention en matière de droits et libertés avec les tribunaux. Le pouvoir politique (c'est-à-dire le pouvoir exécutif et le pouvoir législatif) a perdu de sa capacité d'agir sans entraves autres que celles qu'il acceptait lui-même de se donner en ces domaines au profit du pouvoir judiciaire. L'adoption de la Charte a profondément modifié le visage du droit et de la politique au Canada. Les juges sont devenus des acteurs sociaux incontournables qui ont à se prononcer sur des enjeux jadis réservés aux débats politiques (Woehrling, 2005: 81-118). Ils ont la capacité et le pouvoir juridique de s'opposer à la volonté des élus lorsqu'ils constatent que les dispositions législatives contestées contreviennent au partage des pouvoirs ainsi qu'aux droits et libertés prévus à la constitution.

Depuis 1982, le rôle des tribunaux canadien en matière d'élaboration du droit s'est donc considérablement accru. Les tribunaux ne sont plus contraints d'accepter le caractère définitif des mesures décidées par le législateur. Ils ont le pouvoir de déclarer illégal un texte législatif ou une mesure qui ne respecte pas la Charte. Il y a donc une nouvelle dynamique qui caractérise les relations entre d'une part les tribunaux et d'autre part le Parlement et les assemblées législatives. Il y a maintenant un processus qui nous met en présence d'un dialogue permettant de valider ou d'invalider une loi selon le partage des pouvoirs et des compétences et selon les droits et libertés mentionnés à la Charte. La souveraineté parlementaire au Canada n'est donc plus une puissance illimitée. À la suite de l'adoption de la Charte, en 1982, le Canada est passé, d'une certaine manière, de l'ère de l'État légal à celui de l'État de droit.

Il y a une différence importante entre l'État légal et l'État de droit. Dans le premier cas, le pouvoir exécutif, l'administration et la justice sont soumis au respect de la loi votée par le Parlement, loi qui, comme expression de la volonté générale, est incontestable. L'État légal fait donc référence à une instance étatique (le pouvoir législatif) posée en tant que lieu d'expression de la volonté générale. Pour ce qui est du deuxième, l'État de droit, il ne peut pas être l'État de n'importe quelle loi. Les lois doivent être soumises au respect de normes qui leur sont supérieures et qui fondent en conséquence un contrôle possible des lois. Les lois adoptées doivent respecter la Constitution, les traités et les conventions internationales relatives aux droits de l'homme. D'un point de vue théorique, l'État de droit n'est pas l'État de n'importe quel droit (ou de n'importe quelle loi). Il s'agit plutôt d'un droit (ou de lois) qui est sous-tendu par un ensemble de valeurs et de principes. L'État de droit renvoie très explicitement depuis le lendemain de la Deuxième Guerre mondiale à la démocratie libérale et aux droits de l'homme. Quoi qu'on dise, dans les faits, les États qui se réclament de l'État de droit n'adoptent pas toujours une pratique exemplaire. Il y a souvent, de la part des détenteurs de pouvoir au sein des États qui se réclament de l'État de droit, des défaillances, des décisions contradictoires ou des gestes de mépris à l'endroit de l'État de droit²

2. Sur la notion d'État légal, voir Carré de Malberg (2004: 23-25); sur la notion d'État de droit, voir Perrier (2000: 6-9 et 18-23). 
Puisqu'il appartient aux juges, dans un État de droit, d'interpréter une disposition législative quand survient un différend en matière de droits fondamentaux, examinons si les juges de la Cour suprême du Canada ont adopté une interprétation qui allait dans le sens de l'esprit qui semblait habiter les rédacteurs de la Charte quand ils se sont prononcés sur l'étendue et la portée du droit d'association en matière de droits syndicaux. Mentionnons à ce sujet que, dans l'esprit des rédacteurs de la Charte, il semblait aller de soi que la liberté d'association (vue sous l'angle de la liberté d'association syndicale) incluait également le droit de négociation collective. De plus, les droits reconnus dans les accords internationaux devaient trouver une application concrète autant dans les lois que dans la Charte.

Des débats parlementaires portant sur la Charte, deux déclarations méritent d'être reproduites. La première est celle de Robert Kaplan (ministre de la Justice intérimaire à l'époque). Au sujet de la liberté d'association syndicale et du droit de négociation collective, celui-ci déclare:

Pour ce qui est de la proposition d'inclure un article qui traite tout particulièrement de la liberté d'association et du droit de négociation collective, nous pensons que ces deux points sont couverts par les termes «liberté d'association», qui figurent dans la charte. En créant un article à part qui traiterait du droit à la négociation collective, cela pourrait amoindrir l'importance des autres types d'associations, comme les associations religieuses, fraternelles ou communautaires. (Cité par Bendel, 1986:324)

La deuxième déclaration vient du ministre de la Justice de l'époque, Jean Chrétien; elle porte sur le sens de la liberté d'association en lien avec les accords internationaux dont le Canada est signataire. À ce sujet, M. Chrétien précise: «Je crois en effet que les droits que nous avons reconnus dans les accords internationaux devraient aussi se retrouver dans nos lois ou dans cette charte des droits.» (Cité par Bendel, 1986:324) Jusqu'à quel point les juges de la Cour suprême du Canada ont-ils adopté une interprétation qui allait dans le sens expressément mentionné par MM. Kaplan et Chrétien? C'est à cette question que nous allons tenter de répondre ici.

\section{De la trilogie de 1987 à l'arrêt Health Services and Support (en 2007) ou d'une vision majoritaire fort restrictive de la liberté d'association à son affirmation majoritaire positive}

Depuis l'adoption de la Charte en 1982, les juges de la Cour suprême du Canada ont eu à se demander à plus d'une reprise si la liberté d'association intègre non seulement le droit à l'organisation et à l'affiliation syndicales, mais aussi le droit des membres de négocier leurs conditions de travail et de recourir, au besoin, à la grève lors d'une négociation collective. Durant cette période qui s'échelonne sur deux décennies, on peut distinguer trois tendances interprétatives: une première qu'on peut qualifier de restrictive au sens où la liberté d'association se voyait conféré un caractère purement formel, légaliste et individualiste; une deuxième un peu plus ouverte à la dimension collective de la liberté d'association; et une troisième où la liberté d'association inclut aussi, en matière de droit syndical, le droit à la négociation. 


\section{La période dite restrictive}

La première décision de la Cour suprême du Canada relative à la portée et à l'étendue de la liberté d'association (alinéa 2 d) de la Charte a été rendue en 1987 dans l'affaire Re: Public Service Employee Relations Act (Alberta) (RCS, 1987). Trois lois (la Public Service Employee Relations Act, la Labour Relations Act et la Police Officers Collective Bargaining Act) étaient contestées par les organisations syndicales parce qu'elles allaient, selon ces dernières, à l'encontre de la liberté d'association garantie par la Charte. La Cour suprême avait le choix d'endosser les engagements internationaux du Canada en matière de liberté d'association et de donner suite aux déclarations de MM. Kaplan et Chrétien ou de souscrire à la position des tribunaux de l'Alberta selon laquelle la liberté d'association en matière syndicale ne s'accompagne pas du droit à la négociation et du droit à la grève. Le 9 avril 1987, la Cour suprême a fait son choix. Sa décision fut partagée. Les juges majoritaires concluaient que les droits de négocier collectivement les conditions de travail et de faire grève n'étaient pas inclus dans la liberté d'association (RCS, 1987: 141). Les juges du camp majoritaire sont restés silencieux en regard des engagements internationaux du Canada en matière de droits fondamentaux. Ils épousèrent une définition restrictive de la liberté d'association, mentionnant que celle-ci n'incluait pas de droits qu'une personne ne détient pas individuellement. Ils soulignèrent de plus que ni la négociation collective des conditions de travail ni la grève n'étaient des droits fondamentaux au Canada ${ }^{3}$ (RCS, 1987: 181).

Pour le juge McIntyre, la liberté d'association désigne d'abord le droit de se joindre à d'autres pour se livrer à des activités légitimes communes et de former et de maintenir des associations qui poursuivent de tels buts. Ce droit garantit l'exercice collectif des droits constitutionnels individuels et permet à un individu de poser des actions, de concert avec d'autres, que cet individu pourrait légitimement poser seul (RCS, 1987: 176). Dans son analyse portant sur l'étendue de l'alinéa 2 d), le juge McIntyre accepte la perspective américaine suivant laquelle «c'est l'individu qui constitue l'intérêt ultime de l'ordre social. Ses intérêts et ses droits sont prépondérants » (Emerson, cité par McIntyre dans RCS, 1987:156). Il s'ensuit, selon cette vision de la liberté d'association, que celle-ci ne peut être considérée comme un droit collectif.

Dans cette cause, le juge en chef Dickson inscrit une dissidence dans laquelle il analyse le contenu des engagements internationaux du Canada en matière de liberté d'association et de droits syndicaux. Selon lui, il «existe un consensus manifeste au sein des organismes décisionnels de l'O.I.T. [Organisation internationale du travail] suivant lequel la Convention $n^{\circ} 87$ ne se borne pas uniquement à protéger la formation des syndicats mais protège leurs activités fondamentales, soit la négociation collective et le droit de grève» (RCS, 1987: 72). Au sujet du rôle du droit international dans l'interprétation de la Charte, le juge Dickson écrit:

3. Parlant du droit de grève, le juge McIntyre précise : «On ne peut dire qu'il soit devenu à ce point partie intégrante de nos traditions sociales et historiques au point d'acquérir le statut d'un droit immuable et fondamental, fermement enraciné dans nos traditions et dans notre philosophie politique et sociale. Il n'existe donc aucun motif, comme on le propose dans la quatrième conception de la liberté d'association, de présumer de l'existence d'un droit de grève.» (RCS, 1987: 181) 
Le droit international nous donne un bon aperçu de la nature et de la portée de la liberté d'association des travailleurs. Depuis la fin de la Deuxième Guerre mondiale, la protection des droits et libertés fondamentaux collectifs et individuels est devenue une question d'intérêt international. Il existe maintenant un droit international des droits de la personne constitué d'un ensemble de traités (ou conventions) et de règles coutumières, en vertu duquel les nations du monde se sont engagées à adhérer aux normes et aux principes nécessaires pour assurer la liberté, la dignité et la justice sociale à leurs ressortissants. La Charte est conforme à l'esprit de ce mouvement international contemporain des droits de la personne et elle comporte un bon nombre des principes généraux et prescriptions des divers instruments internationaux concernant les droits de la personne. Les diverses sources du droit international des droits de la personne - les déclarations, les pactes, les conventions, les décisions judiciaires et quasi judiciaires des tribunaux internationaux, et les règles coutumières - doivent, à mon avis, être considérées comme des sources pertinentes et persuasives quand il s'agit d'interpréter des dispositions de la Charte [...]

En outre, le Canada est partie à plusieurs conventions internationales sur les droits de la personne qui comportent des dispositions analogues ou identiques à celles de la Charte. Le Canada s'est donc obligé internationalement à assurer à l'intérieur de ses frontières la protection de certains droits et libertés fondamentaux qui figurent dans la Charte. Les principes généraux d'interprétation constitutionnelle requièrent que ces obligations internationales soient considérées comme un facteur pertinent et persuasif quand il s'agit d'interpréter la Charte [...] Je crois qu'il faut présumer, en général, que la Charte accorde une protection au moins aussi grande que celle qu'offrent les dispositions similaires des instruments internationaux que le Canada a ratifiés en matière de droits de la personne. (RCS, 1987:57 et 59)

Dans sa dissidence, le juge Dickson identifie les divers instruments ratifiés par le Canada qui traitent de la liberté d'association: le Pacte international relatif aux droits économiques, sociaux et culturels, le Pacte international relatif aux droits civils et politiques et la Convention sur la liberté syndicale et la protection du droit syndical, 1948 ( $\mathrm{n}^{\circ} 87$ ) de l'OIT. Au sujet de la Convention $n^{\circ}$ 87, il mentionne que les organes décisionnels de l'OIT considèrent que la liberté de former et d'organiser des syndicats inclut celles de négocier collectivement des conditions de travail et de faire grève. Il précise aussi le fait que le Comité de la liberté syndicale et le Conseil d'administration de l'OIT ont étudié un certain nombre de plaintes formulées contre le Canada par des syndicats canadiens à partir de la Convention $n^{\circ} 87$. Certaines de celles-ci avaient pour effet de contester les dispositions des trois lois de l'Alberta qui étaient l'objet du pourvoi devant la Cour suprême. Le juge Dickson rappelle que les instances de l'OIT avaient condamné unanimement le Canada, en ce que les prohibitions du droit de grève contenues dans ces lois étaient beaucoup trop larges, de telles restrictions ne devant s'appliquer, selon les instances de l'OIT, qu'aux employés exerçant vraiment des services essentiels (Trudeau, 2001: 188). De plus, il réprouve «la nature essentiellement formelle» de «la définition constitutive» de la liberté d'association retenue par ses collègues majoritaires. À ce sujet il mentionne: «Si la liberté d'association ne protège que la réunion de personnes à des fins communes, mais non l'exercice des activités mêmes pour lesquelles l'association a été formée, alors cette liberté est effectivement légaliste, parcimonieuse et voire même insipide.» (RCS, 1987: 81) 
En 1990, dans l'affaire Institut professionnel de la fonction publique du Canada c. Commissaire des Territoires du Nord-Ouest et Northwest Territories Public Services Association et al. (RCS, 1990b: 367), les juges de la Cour suprême réitèrent les conclusions de la jurisprudence établie en 1987. Dans ce cas-ci, il s'agit pour les juges de décider si une loi des Territoires du Nord-Ouest va à l'encontre de la liberté d'association garantie par l'alinéa 2 d) de la Charte. En vertu de cette loi, une association de salariés (de fonctionnaires) est autorisée à négocier collectivement les conditions de travail avec le gouvernement en autant que l'association soit constituée en personne morale par une loi qui l'autorise à négocier. Une telle obligation a pour effet de laisser entre les mains du gouvernement l'entière discrétion quant à l'accréditation de l'association de salariés représentant ses fonctionnaires. Les juges de la Cour suprême confirment la validité constitutionnelle de cette loi, la liberté d'association selon eux ne protégeant que la liberté des individus de s'associer entre eux, non les droits collectifs rattachés au syndicat. Cette décision a comme conséquence de préciser que l'alinéa 2 d) n'a pas pour effet d'interdire l'adoption de lois imposant des conditions précises à l'octroi d'une accréditation syndicale, même si cela a pour résultat de priver une association de la possibilité de représenter un groupe de salariés donné (Notebaert, 2007: 7). Ici aussi, les juges ne tiennent pas compte des instruments internationaux pertinents ratifiés par le Canada. Pour eux, la liberté d'association a pour effet de protéger uniquement «la liberté de travailler à la constitution d'une association, d'appartenir à une association, de la maintenir et de participer à ses activités licites sans faire l'objet d'une peine ou de représailles » (RCS, 1987: 143) et sans être exposé à quelque ingérence de l'employeur dans la formation de cette association.

Mentionnons-le, les juges de la Cour suprême du Canada ne se sont pas toujours montrés hostiles à l'idée d'appuyer leurs décisions en tenant compte des instruments internationaux ratifiés par le Canada. En 1997, dans l'affaire Godbout c. Ville de Longueuil (RCS, 1997: 69), les juges avaient à décider si l'obligation de résider dans les limites de la ville pour y détenir un emploi de fonctionnaire enfreignait le droit à la liberté garanti par l'article 7 de la Charte. Trois juges décident alors d'inclure dans ce droit à la liberté celui de choisir son lieu de résidence. En appui à leur décision, ils invoquent le Pacte international relatif aux droits civils et politiques (Pacte ratifié par le Canada) selon lequel à l'article 12 le droit de choisir sa résidence est protégé. Au nom de ces trois juges, le juge La Forest précise:

Comme notre Cour a reconnu la valeur de persuasion des pactes internationaux dans la définition de la portée des droits garantis par la Charte (références à l'arrêt Slaight Communications et à l'affaire Re: Public Service Employee relations Act (Alberta), je considère que l'article 12 renforce ma conclusion voulant que le droit de décider où établir sa demeure fasse partie de la sphère irréductible d'autonomie personnelle protégée par la garantie de liberté énoncée à l'article 7. (RCS, 1997:69)

En 1999, dans l'affaire Delisle c. Procureur du Canada et al. (RCS, 1999b: 989), les juges de la Cour suprême décident que la liberté d'association garantie à l'alinéa 2 d) de la Charte n'a pas pour effet de contraindre le législateur à assurer à un groupe de salariés un régime syndical particulier d'association. Il est donc décidé que l'exclusion des membres de la Gendarmerie royale du Canada (GRC) du régime du Code canadien du travail et de la Loi sur les relations de travail dans la fonction publique ne va pas à l'encontre de la Charte. Ici aussi, les juges majoritaires rendent cette décision sans faire allusion aux instruments internationaux pertinents 
ratifiés par le Canada. Les juges minoritaires se donnent la peine de faire allusion aux instruments internationaux ratifiés par le Canada. Selon eux, l'objet même de la loi, qui avait pour objectif d'empêcher les membres de la GRC de former une association d'employés, constituait une dérogation manifeste de la liberté d'association garantie par l'alinéa 2 d) de la Charte. À la base de leur raisonnement, ils invoquent divers instruments internationaux ratifiés par le Canada qui reconnaissent tous «la liberté fondamentale des employés de s'associer pour défendre leurs intérêts à titre d'employés» (RCS, 1999b: 71).

En 1999, les juges de la Cour suprême du Canada rendent une décision qui aura un impact majeur quant au lien à établir entre le droit interne et la portée des instruments internationaux ratifiés par le Canada. Dans l'affaire Baker (RCS, 1999a: 817), il s'agissait de définir les critères applicables au contrôle judiciaire d'une décision de nature discrétionnaire rendue par le ministre canadien de l'Emploi et de l'Immigration. Les juges de la Cour avaient à se demander si le ministre était autorisé, en vertu de la Loi sur l'immigration et de la réglementation applicable, à faciliter l'admission au Canada d'une personne pour des raisons humanitaires. Il s'agissait donc de vérifier l'étendue du pouvoir discrétionnaire d'ordre humanitaire du ministre. À ce sujet, dans la décision majoritaire, les juges mettent de l'avant le principe suivant: «il faut que le pouvoir discrétionnaire soit exercé conformément aux limites imposées dans la loi, aux principes de la primauté du droit, aux principes du droit administratif, aux valeurs fondamentales de la société canadienne, et aux principes de la Charte» (RCS, 1999a: 56). En lien avec les «valeurs fondamentales de la société canadienne», les juges concluent que, dans une telle situation discrétionnaire d'ordre humanitaire, le ministre doit porter une attention particulière aux besoins et aux intérêts des enfants. À ce sujet, pour les juges de la Cour, il apparaît clairement que les instruments internationaux ratifiés par le Canada (bien que ne faisant pas partie en tant que tels du droit canadien en raison du caractère dualiste de l'État canadien) doivent constituer un élément important dans la détermination des valeurs de la société canadienne (Trudeau, 2001: 192).

Ce bref survol de la jurisprudence de la Cour suprême du Canada démontre que jusqu'à tout récemment les instruments internationaux ratifiés par le Canada en matière de travail ne trouvaient pas toujours écho chez la majorité des juges de la Cour suprême du Canada. Les obligations internationales du Canada n'ont pas été suffisantes pour amener les juges de la Cour suprême à incorporer le droit de négociation collective et le droit de grève dans la notion de liberté d'association lorsqu'il s'agissait d'interpréter l'alinéa 2 d) de la Charte. Par contre, comme on vient de le voir avec les décisions Godbout et Baker, les juges de la Cour suprême du Canada se sont montrés ouverts à l'idée d'appuyer solidement leur décision sur les ententes internationales ratifiées par le Canada pour donner une interprétation large et libérale des droits et libertés prévus à la Charte. Dans l'ensemble, il se dégage de ce premier survol des décisions de la Cour suprême du Canada de 1987 à 2000 que nous sommes face à une conception restrictive de la liberté d'association. Plus spécifiquement, cette conception formelle est centrée sur l'individu, dans la plus pure lignée du libéralisme classique dont se réclament les principaux droits de la Charte, et repose sur la prémisse que l'objet premier de l'alinéa 2 d) consiste à assurer l'épanouissement individuel au moyen de relations avec autrui. C'est du moins ce qui nous semble transparaître de l'ensemble de la jurisprudence examinée jusqu'à maintenant. 


\section{Une période marquée par la reconnaissance de la dimension collective de la liberté d'association}

Dans l'arrêt Dunmore (RCS, 2001: 1016), prononcé en 2001, les juges de la Cour suprême du Canada acceptent d'accorder un caractère un peu plus collectif à la liberté d'association. Les juges étaient appelés à statuer sur la légalité de la loi ontarienne de 1995. Cette loi avait pour effet d'en abroger une autre - adoptée deux ans plus tôt - selon laquelle les travailleurs agricoles de la province avaient le droit à la syndicalisation. La loi abrogative fut déclarée inconstitutionnelle (Brunelle et Verge, 2003: 721). Les juges de la Cour suprême conviennent que la liberté d'association n'exige pas seulement du législateur qu'il s'abstienne de toute intervention portant atteinte à une activité associative protégée, mais que cette liberté entraîne également, «dans des contextes exceptionnels», une obligation positive de sa part si son inaction a un «effet substantiel sur l'exercice d'une liberté constitutionnelle» (RCS, 2001: 22). Pour les juges de la Cour suprême, une mesure étatique dont la portée se révèle trop limitative peut aller à l'encontre de la liberté d'association dans la mesure où le législateur «orchestre, encourage ou tolère d'une manière substantielle la violation de libertés fondamentales » (RCS, 2001:26). Plus important encore, ces juges reconnaissent que la liberté d'association peut protéger des activités «de nature collective» «qui ne peuvent, pour une raison ou une autre, être considérées comme des activités licites» (RCS, 2001: 16) d'une personne seule. Précisant que «les individus ne s'associent pas simplement pour la force du nombre, mais aussi parce qu'une collectivité peut incarner des objectifs qui n'existent pas au niveau individuel», les juges mentionnent:

étant donné que les besoins et priorités des syndicats tendent à se distinguer de ceux de leurs membres individuels, ils ne peuvent fonctionner si la loi protège exclusivement ce qui pourrait être des «activités licites d'un individu». La loi doit plutôt reconnaître que certaines activités syndicales - les revendications collectives auprès de l'employeur, l'adoption d'une plate-forme politique majoritaire, le regroupement en fédérations syndicales - peuvent être au cœur de la liberté d'association même si elles ne peuvent exister au niveau individuel [...] Cela veut dire simplement que certaines activités collectives doivent être reconnues pour que la liberté de constituer et de maintenir une association ait un sens. (RCS, 2001:17)

Dans la foulée de la décision Baker, on décèle ici aussi une nouvelle appréciation de la pertinence du droit international. Dans la décision Dunmore, les juges élargissent la portée de la liberté d'association en acceptant que l'alinéa 2 d) a pour effet de protéger certaines activités qui n'ont de sens qu'au niveau associatif. Ils écrivent même que la collectivité peut «incarner des objectifs qui n'existent pas au niveau individuel» (RCS, 2001: 16). Ainsi donc, sans admettre que le droit de négociation collective et le droit de grève font partie de ces activités collectives qui sont protégées par la Charte, les juges de la Cour suprême décident d'invalider une loi qui avait pour effet d'exclure du droit à la syndicalisation certains travailleurs particulièrement vulnérables. C'est en se référant à la jurisprudence du Comité des libertés syndicales du Bureau international du travail et aux dispositions de la Convention $n^{\circ} 87$ qu'ils jugent que l'exclusion des travailleurs agricoles du régime de rapports collectifs de travail mis en place par le gouvernement ontarien avait pour effet de porter atteinte à leur liberté fondamentale de s'organiser (Notebaert, 2007:7-8). La décision Dunmore vient préciser que le pouvoir législatif ne peut pas interdire aux travailleurs agricoles de l'Ontario le droit de s'associer (donc le droit 
de former un syndicat). Toute interdiction de ce genre, dans la Loi de 1995 sur les relations de travail de l'Ontario, a pour effet de faire de la liberté d'association un «droit fictif» (RCS, 2001: 146). «Dans une démocratie constitutionnelle, il ne suffit pas de protéger les libertés fondamentales contre les mesures de l'État: il faut aussi leur assurer un "espace vital” » (RCS, 2001: 148), écrivent les juges dans la décision Dunmore.

Pour les juges de la Cour suprême du Canada, à partir de l'arrêt Dunmore, la liberté d'association s'élargit et inclut désormais «le droit de se syndiquer [...] avec les garanties jugées essentielles à son exercice véritable, comme la liberté de se réunir, de participer aux activités légitimes de l'association et de présenter des revendications, et la protection de l'exercice de ces libertés contre l'ingérence, les menaces et la discrimination» (RCS, 2001: 67) .

Il semble donc qu'en droit canadien, jusqu'à la décision Dunmore, contrairement au droit international, la liberté syndicale et la liberté d'association s'avèrent être deux concepts distincts. Leur contenu n'est pas superposable. Le premier préserve le droit de constituer un syndicat et les grands modes traditionnels d'action (la négociation collective et le recours à la grève), le deuxième, selon l'interprétation de la Cour suprême du Canada, se borne à protéger les aspects constitutifs de l'association sans pour autant étendre sa protection à ses activités fondamentales (droit à la négociation collective et droit à la grève) (Brunelle et Verge, 2003: 746). Il y a néanmoins dans la décision Dunmore une ouverture en ce sens.

\section{Quand la liberté d'association s'accompagne d'une reconnaissance du droit à la négociation}

Dans une décision rendue le 8 juin 2007, les juges de la Cour suprême du Canada reconnaissent les liens intrinsèques qui existent entre la liberté d'association (mentionnée à l'alinéa $2 \mathrm{~d}$ ) de la Charte) et le droit de négociation en matière de droit syndical. La position prise par la Cour suprême en 1987, selon laquelle la liberté d'association ne protégeait pas le droit de négocier, a finalement été écartée (Coutu, 2007: b5). Il existe donc maintenant, en droit canadien, une liberté constitutionnelle de négocier collectivement ses conditions de travail.

Notre conclusion que l'al. 2 d) de la Charte protège le processus de négociation collective repose sur quatre prémisses. Premièrement, l'examen de la jurisprudence de la Cour sur l'al. 2 d) révèle que les raisons invoquées par le passé pour expliquer que le droit à la liberté d'association ne s'étendait pas à la négociation collective ne valent plus. Deuxièmement, une interprétation de l'al. 2 d) qui exclut la négociation collective de son champ d'application ne se concilie pas avec le fait que le Canada a toujours reconnu l'importance de ce processus en matière de liberté d'association. Troisièmement, la négociation collective fait partie intégrante de la liberté d'association selon le droit international, qui peut inspirer l'interprétation des garanties de la Charte. Enfin, interpréter l'al. 2 d) comme comprenant le droit de négociation collective s'intègre dans la logique, voire la défense, des autres droits, libertés et valeurs consacrés par la Charte (CSC, 2007: 20).

Si l'interprétation donnée de l'alinéa 2 d) de la Charte par les juges de la Cour suprême depuis 1987 était restrictive par rapport à la jurisprudence internationale, l'interprétation qu'elle en fait maintenant nous permet d'affirmer que le droit canadien interne accorde une certaine protection au droit des travailleurs de constituer 
une organisation syndicale de leur choix, de la maintenir et d'y appartenir. L'alinéa 2 d) de la Charte protège donc deux des trois composantes d'un véritable régime de liberté syndicale ${ }^{4}$ (la liberté d'association et le droit à la négociation). Pour les juges, l'alinéa 2 d) doit maintenant être interprété «comme ayant pour effet de protéger le droit d'employés de s'associer en vue d'atteindre des objectifs relatifs au milieu de travail par un processus de négociation collective» (CSC, 2007: 87). Cet alinéa vise non seulement «les activités individuelles exercées collectivement, mais aussi les activités associatives elles-mêmes»(CSC, 2007: 89). Avec cette décision, les juges de la Cour suprême du Canada confirment la justesse des principes énoncés dans l'affaire Dunmore et réfutent formellement les arrêts Renvoi relatif à l'Alberta et Institut professionnel qui avaient pour effet d'exclure le droit à la négociation collective de toute protection constitutionnelle (Notebaert, 2007: 12-16). C'est un changement de paradigme majeur qui illustre, d'une part, la discrétion - encadrée par le droit - dans l'interprétation d'un concept aussi flou que «liberté d'association » et, d'autre part, une sensibilité plus grande aux dimensions collectives des droits et libertés constitutionnels, même s'ils sont formulés en termes individuels. On retrouve la même tendance en droits linguistiques (Mahe c. Alberta) (RCS, 1990a: 342). Rien cependant ne garantit la pérennité de cette approche car, dans un régime de common law, la discrétion judiciaire autorise des bifurcations jurisprudentielles fondées sur des arguments et des faits différents de ceux des décisions antérieures. Ainsi par exemple, l'arrêt Chaoulli (RCS, 2005: 791) manifeste, en rapport avec l'accès aux soins de santé, une propension aux libertés individuelles plutôt qu'aux dimensions collectives engendrées par un régime public de soins de santé.

Cette nouvelle tendance interprétative de la portée de la liberté d'association en matière de droit du travail peut-elle avoir pour effet de mettre un terme à «l'exceptionnalisme permanent» qui semble être la tendance forte lors des négociations dans les secteurs public et parapublic au Québec?

\section{La fin de l'exceptionnalisme permanent?}

Pour ce qui est du droit à la négociation des rapports collectifs de travail, un survol historique du déroulement des négociations dans les secteurs public et parapublic peut s'avérer intéressant ici. Mentionnons que, de 1964 à 2005, nous dénombrons plus de seize grands rendez-vous de négociations dans les secteurs public et parapublic entre le gouvernement du Québec et les organisations syndicales. Ces négociations ont eu pour objet soit la négociation de l'ensemble des dispositions de la convention collective ou encore la négociation de certains aspects de la convention collective (la rémunération surtout). Les négociations collectives dans les secteurs public et parapublic n'ont pas toujours suivi un parcours exemplaire et conforme à la loi. Elles ont souvent donné lieu à une négation du droit de négociation ou (et) du droit de grève. La reconnaissance constitutionnelle d'un droit de négociation collective retire donc de l'arène politique (sous réserve des limites prévues aux articles 1 et 33 de la Charte) ce qui, historiquement, en avait fait partie. Qu'on en juge par le récit historique qui suit (Perrier, 1992;1999; 2007).

4. Pour Guy Caire (1976: 15), un véritable régime de liberté syndicale comporte au moins les trois éléments suivants: la liberté d'association, la libre négociation et le droit de grève. 


\section{Les négociations dans les secteurs public et parapublic au Québec de 1964 à 1985}

De l'adoption du Code du travail (en 1964) et de la Loi de la fonction publique (en 1965), à l'adoption de la Loi sur le régime de négociation des conventions collectives dans les secteurs public et parapublic (L.R.Q., chap. R8.2) (en 1985), six rondes de négociations ont eu lieu entre le gouvernement et les organisations syndicales de ces secteurs : 1) 1964 à 1967;2) 1968;3) 1971; 4) 1976; 5) 1979; et 6) 1982-1983. Une seule de ces négociations, celle de 1968, n'a pas donné lieu à l'adoption d'une loi spéciale. Toutes les autres ont fait l'objet de mesures dilatoires ou exceptionnelles interdisant ou reportant l'exercice du droit de grève, imposant un retour au travail, décrétant les conditions de travail des salariés et salariées syndiqués, freinant ou réduisant les hausses de salaires. Les lois spéciales adoptées au cours des négociations entre l'État et les personnes salariées des secteurs public et parapublic sont les suivantes:

- Le projet de loi 25 (Loi assurant le droit de l'enfant à l'éducation et instituant un nouveau régime de convention collective dans le secteur scolaire (S.Q.1966-67, c. 63)), en 1967, qui a pour effet de retirer aux enseignants et enseignantes leur droit de grève et de créer une structure de négociation centralisée.

- $\quad$ Le projet de loi 19 (Loi assurant la reprise des services dans le secteur public (L.Q.1972, c. 7)), en 1972, qui force le retour au travail de tous les grévistes des secteurs public et parapublic et qui exige la conclusion d'une entente collective pour éviter qu'il n'y ait un décret tenant lieu de convention collective.

- $\quad$ Le projet de loi 64 (Loi concernant les mesures anti-inflationnistes (L.Q. 1975, c. 16)), en 1975, qui a pour effet de limiter les hausses de salaires. (Le règlement conclu entre le gouvernement et les organisations syndicales dépassera les limites fixées par cette loi.)

- Le projet de loi 23 (Loi concernant le maintien des services dans le domaine de l'éducation et abrogeant une disposition législative (L.Q. 1976, c. 38)), en 1976, qui défend aux salariés d'une commission scolaire ou d'un cégep tout ralentissement ou diminution de l'activité normale de travail pour une période de 80 jours.

- Le projet de loi 61 (Loi concernant les services de santé dans certains établissements (L.Q.1976, c. 29)), en 1976, qui impose d'autorité un retour au travail aux infirmières en grève et interdit tout ralentissement et diminution de l'activité normale de travail. La loi décrète également leurs conditions de travail.

- $\quad$ Le projet de loi 62 (Loi sur les propositions aux salariés des secteurs de l'éducation, des affaires sociales et de la fonction publique (L.Q. 1979, c. 50)), en 1979, qui, sans abolir le droit de grève, en reporte l'exercice.

- Les projets de lois 68 (Loi modifiant diverses dispositions législatives concernant les régimes de retraite (L.Q. 1982, c. 33)), 70 (Loi concernant la rémunération dans le secteur public (L.Q. 1982, c. 45)), 105 (Loi concernant les conditions de travail dans le secteur public (L.Q. 1982, c. 45)) et 111 (Loi assurant la reprise des services dans les collèges et les écoles du 
secteur public (L.Q.1983, c. 1)), adoptés en 1982 (pour les trois premières) et en 1983 (pour la dernière). Le projet de loi 68 a pour effet de réduire unilatéralement certains avantages du régime de retraite des employés et des employées de l'État, de l'éducation et des affaires sociales. Le projet de loi 70 prolonge les conventions collectives de trois mois et réduit les traitements de $19,5 \%$ pour cette période. Cette loi a pour effet, avant même l'ouverture officielle des négociations, de retarder la possibilité d'exercer le droit de grève. Alors qu'aucune grève n'est en cours, en décembre 1982, le gouvernement du Québec fait adopter par l'Assemblée nationale le projet de loi 105, qui a pour effet de décréter les conditions de travail et de rémunération du personnel salarié syndiqué des secteurs public et parapublic et leur retire le droit de grève jusqu'aux prochaines négociations (les décrets venant à échéance le 31 décembre 1985). Devant la grève dite «illégale» des enseignants et des enseignantes, le gouvernement adopte le projet de loi 111, qui suspend l'application des droits prévus aux chartes et force les salariés syndiqués à retourner au travail au plus tard le 17 février 1983, et ce, jusqu'au 31 décembre 1985.

Ce rapide survol des rondes de négociation dans les secteurs public et parapublic montre que ces négociations ont été mouvementées. À la suite de l'affrontement de 1982-1983, le gouvernement de René Lévesque décidera de modifier en profondeur le régime de négociation dans les secteurs public et parapublic et établira de nouvelles règles du jeu en procédant, en 1985, à l'adoption du projet de loi 37 (Loi sur le régime de négociation des conventions collectives dans les secteurs public et parapublic (L.R.Q., chap. R8.2)).

Sans entrer dans les détails de cette loi, mentionnons que la répartition des matières négociables entre le niveau provincial et les niveaux régional et local y est dessinée: les matières négociables au niveau provincial sont substantiellement réduites; un nouveau mode de fixation des salaires et des échelles de salaires est établi; ces matières sont négociables pour la première année des conventions collectives seulement et, en ce qui a trait aux deux autres années, elles seront fixées par règlement. Cette loi modifie le Code du travail en matière de services essentiels. Il précise le pourcentage de personnel salarié à maintenir par quart de travail (pourcentage qui varie de $55 \%$ à $90 \%$ selon les services des établissements hospitaliers, de santé et de services sociaux). Les décisions du Conseil des services essentiels sont impératives. Le Conseil peut ordonner ou interdire de faire quelle que chose que ce soit à toute personne. Aucune grève n'est possible dans les établissements où il n'existe pas de liste de services essentiels approuvée par le Conseil. Pour ce qui est des salaires, le droit de grève est possible pour la première année de la convention collective seulement.

Le gouvernement de Robert Bourassa fait adopter, en 1986, le projet de loi 160 (Loi assurant le maintien des services essentiels dans le secteur de la santé et des services sociaux (L.Q. 1986, c. 74)). Cette loi vise à encadrer de façon très stricte l'exercice de la grève dans les secteurs mentionnés dans le titre de la loi.

\section{Les négociations depuis l'adoption des lois 37 et 160}

Depuis l'adoption du projet de loi 37 en 1985, dix négociations ont eu lieu entre l'État et le personnel syndiqué des secteurs public et parapublic: 1986, 1989, 1991, 1992, 1993, 1995, 1996-1997, 1998-1999, 2002 et 2003 à 2005. La nouvelle loi régissant 
la négociation dans les secteurs public et parapublic prévoit que seule la première année de la convention collective peut faire l'objet d'une négociation en ce qui concerne les salaires, les deux suivantes devant faire l'objet d'un règlement adopté par le gouvernement. Fait important à souligner, ces négociations se sont toutes conclues dans un cadre fort différent de celui prévu dans la loi. Qu'on en juge par ce qui suit:

- En 1986, le gouvernement du Québec convient d'une convention collective pour une durée de trois ans, accordant des augmentations de salaire de l'ordre de 3,5\% (le $1^{\text {er }}$ janvier 1986), de $4 \%$ (le $1^{\text {er }}$ janvier 1987 ) et de $4,5 \%$ (le $1^{\text {er }}$ janvier 1988). À cela s'ajoute une indexation pouvant aller jusqu'à $1 \%$, si l'indice des prix à la consommation en décembre 1987 dépasse de plus de $4,25 \%$ celui de décembre 1986 . Cette convention collective vient à échéance le 31 décembre 1988.

- Aux négociations de 1989, les augmentations salariales convenues entre les parties en présence sont de l'ordre de $4 \%$ en 1989, de 4,5\% en 1990 et de $4 \%$ en 1991. Les conventions collectives expirent le 31 décembre 1991.

- $\quad$ En avril 1991, une entente de principe, conclue entre le gouvernement et les représentants syndicaux, prévoit une prolongation de six mois de la convention collective (l'échéance des conventions est reportée du 31 décembre 1991 au 30 juin 1992), accompagnée d'une augmentation de $3 \%$ au dernier jour de la convention collective (1 $1^{\mathrm{er}}$ juillet 1992).

- $\quad$ En février 1992, le président du Conseil du trésor invite les organisations syndicales à renoncer à l'entente conclue pour la remplacer par une nouvelle prolongation qui comportera des concessions salariales de la part des salariées et des salariés de l'État. Les parties conviennent en mai 1992 d'une nouvelle prolongation d'un an de la convention collective (du 30 mai 1992 au 30 juin 1993). L'augmentation salariale de $3 \%$ au $1^{\text {er }}$ juillet 1992 est maintenue.

- $\quad$ En juin 1993, le gouvernement adopte une loi spéciale, la Loi concernant les conditions de travail dans le secteur public et le secteur municipal (L.Q. 1993, c. 37) (projet de loi 102), qui décrète une prolongation des conventions collectives du 30 juin 1993 au 30 juin 1995. La masse salariale est amputée de $1 \%$ (ce qui correspond à 2,6 jours de congé sans rémunération pour les années 1993-1994 et 1994-1995). Les clauses normatives sont reconduites pour une période de deux ans. Le gouvernement offre la possibilité de remplacer la coupure salariale de $1 \%$ par d'autres économies dans l'organisation du travail.

- En août 1995 (à quelques semaines du référendum d'octobre) commencent des négociations-marathon en vue du renouvellement des conventions collectives échues depuis juin 1995. Les parties conviennent d'abroger le projet de loi 102 à compter d'octobre 1995 et s'entendent sur les augmentations salariales suivantes: $1 \%$ le $1^{\text {er }}$ mars 1997 et $1 \%$ le $1^{\text {er }}$ mars 1998 . La convention doit venir à échéance le 30 juin 1998.

- Au début de 1997 (en février), le gouvernement rouvre les conventions collectives et fixe des objectifs de résultat. Les salariés syndiqués des secteurs public et parapublic doivent accepter diverses formes de coupures équivalant à $6 \%$ des coûts de main-d'œuvre. L'objectif visé par le gouvernement est la diminution de 15000 postes à temps complet, objectif qu'il parvient 
à imposer aux organisations syndicales en adoptant une loi spéciale, la Loi sur la diminution des coûts de la main-d'œuvre dans le secteur public et donnant suite aux ententes intervenues à cette fin (L.Q. 1997, c. 7) (projet de loi 104).

- En décembre 1999 (après 18 mois de négociations), le front commun syndical (CEQ-CSN-FTQ) obtient une entente négociée d'une durée de quatre ans. Les hausses salariales convenues sont de l'ordre de $9 \%(1,5 \%$ pour $1999 ; 2,5 \%$ par année pour les trois années suivantes).

- Du printemps 2001 au printemps 2003, l'intersyndicale formée de la CSN, la CSQ, la FIIQ, la FTQ et le SFPQ entreprend des travaux visant à réaliser l'équité salariale dans les secteurs public et parapublic au Québec (identification des catégories d'emploi à prédominance féminine, élaboration d'un plan d'évaluation et rédaction d'un questionnaire d'enquête accompagné d'un échantillonnage). En vue de régler le dossier de l'équité salariale, il est même convenu en 2002 de prolonger du 30 juin 2002 au 30 juin 2003 les conventions collectives dans les secteurs public et parapublic. L'entente de prolongation des conventions collectives implique les organisations suivantes: CSQ, FTQ, SFPQ et trois fédérations de la CSN (FSSS, FEESP et FP).

- Lors des négociations qui se dérouleront entre 2003 et 2005, l'équipe ministérielle décide de recourir à la voie autoritaire pour imposer unilatéralement son point de vue. Les négociations se terminent par l'adoption du projet de loi 142 (Loi concernant les conditions de travail dans le secteur public (L.Q. 2005, chap. 43)) qui touche les employés du gouvernement et les salariés travaillant dans les commissions scolaires, les collèges et les établissements du réseau de la santé et des services sociaux. L'objectif de la loi tel que défini à l'article 1 vise deux choses: "assurer la continuité des services publics" et "pourvoir aux conditions de travail des salariés des organismes du secteur public dans le cadre des limites qu'impose la situation des finances publiques». Les conventions collectives sont renouvelées jusqu'au 31 mars 2010. L'annexe 1 fixe la hausse des taux de traitement applicables pour les années 2006 à 2009. Ce taux est établi à $2 \%$ de majoration annuelle. Rien n'est prévu pour les années 2004 et 2005 . Pour ce qui est de la continuité des services, la section IV de la loi (art. 22 à 42) retire le droit de grève que possédaient les salariés et met en place diverses sanctions ayant pour but d'empêcher l'exercice de tout moyen de pression dans les secteurs public et parapublic jusqu'au 31 mars 2010. À cette mesure autoritaire s'ajoute l'adoption, à l'automne 2003, de toute une panoplie de lois antisyndicales qui auront eu pour effet de nuire au déroulement normal de la négociation. Voir à ce sujet: la Loi sur les agences de développement de réseaux locaux de services de santé et de services sociaux - projet de loi 25 (2003, chap. 21); la Loi concernant les unités de négociation dans le secteur des affaires sociales et modifiant la Loi sur le régime de négociation des conventions collectives dans les secteurs public et parapublic - projet de loi 30 (2003, chap. 25); et la Loi modifiant le Code du travail - projet de loi 31 (2003, chap. 26). La loi issue du projet de loi 30 sera déclarée inconstitutionnelle par une juge de la Cour supérieure du Québec le 30 novembre 2007. Rappelons ici que cette loi imposait une restructuration unilatérale des unités syndicales dans les établissements de santé et des services sociaux. Elle avait pour effet de les restructurer 
en quatre catégories distinctes. Elle avait aussi comme objectif de ramener au niveau local la négociation de 26 sujets des conventions collectives sans que les salariés syndiqués puissent recourir à la grève et à un authentique mécanisme d'arbitrage. Dans son jugement, la juge Claudine Roy statue que la loi constitue une atteinte à la liberté d'association (reconnue par la Charte canadienne des droits) et va à l'encontre des obligations internationales contractées par le Canada (Cour supérieure du Québec, 2007).

Malgré le projet de loi 37, les centrales syndicales parviennent donc à négocier avec le gouvernement, à trois reprises, des conventions collectives d'une durée de trois ans (en 1986, en 1989 et en 1995) et, à une occasion, un contrat de travail d'une durée de quatre ans (1998-2002). De plus, à la suite de ces négociations avec le gouvernement, elles obtiennent des prolongations de contrat de travail (en 1991, en 1992 et en 2002). En 1993, cependant, le gouvernement adopte une loi spéciale qui vient mettre un terme à la négociation et, en 1996-1997 (sous la menace d'une loi spéciale), le gouvernement réussit à rouvrir les conventions collectives en imposant une réduction de $6 \%$ des coûts de main-d'œuvre. En décembre 2005, le gouvernement du Québec met fin abruptement à la négociation en interdisant l'exercice de tout moyen de pression et en décrétant, pour une période de six ans, les conditions de travail et de rémunération de ses salariés.

Depuis l'entrée en vigueur du projet de loi 37, on peut dire que les relations de travail dans les secteurs public et parapublic se sont à nouveau terminées par des lois d'exception ou par des reconductions unilatérales de conventions collectives venues à expiration. Les négociations collectives se sont plus souvent qu'autrement conclues à l'extérieur des cadres prévus par la loi, nous permettant dès lors de croire que le régime de négociations dans les secteurs public et parapublic est un authentique régime de négociation «de façade».

Durant les années 1980, Leo Panitch et Donald Swartz (1988) ont forgé l'expression «d'exceptionnalisme permanent» pour rendre compte de la nouvelle dynamique qui semblait caractériser les relations de travail au Canada à la suite de l'adoption de la Loi sur les restrictions salariales du secteur public (S.C. 1980-81-82, c. 122) par le gouvernement de Pierre Elliott Trudeau en 1982 (loi par laquelle le gouvernement canadien a imposé un programme de hausse salariale de 6 et $5 \%$ aux salariés et salariées des fonctions publique et parapublique). Pour ces deux auteurs, cette loi a eu pour effet de mettre un terme à «l'ère de la libre négociation» introduite au Canada vers la fin de la Deuxième Guerre mondiale. C'est du moins ce qu'ils soutiennent: «Developments in the legislative regime governing industrial relations in Canada during the post-controls period hardly suggest a return to the status quo ante but rather a continued movement away from the principles characteristic of the post-war era of "free collective bargaining," and toward the institutionalization of "permanent exceptionalism”.» (1988: 15)

Après une analyse des nombreuses lois spéciales adoptées par les divers gouvernements au Canada, Leo Panitch et Donald Swartz affirment que ces mesures qui se voulaient «spéciales» et «temporaires» durant les années 1960 et 1970 se sont établies comme de véritables normes dans le domaine des relations de travail à partir du début des années 1980. L'exception devenait de plus en plus la règle. Ces nouvelles règles avaient pour effet de codifier le retrait permanent de certains droits comme le droit de grève intimement associé au droit à la négociation. À la lumière de certains jugements récents de la Cour suprême du Canada, une question nouvelle 
se pose: les gouvernements seront-ils toujours autorisés à recourir aussi fréquemment à des mesures autoritaires qui ont pour effet d'empêcher la libre négociation et l'exercice du droit de grève?

\section{Sur l'avenir des négociations ${ }^{5}$ dans les secteurs public et parapublic au Québec}

Manifestement, il est trop tôt pour évaluer l'impact qu'aura l'arrêt Health Services and Support au Canada en matière de négociation collective. Une chose nous paraît certaine, les juges de la Cour suprême semblent convenir que l'interprétation à donner aux droits et libertés fondamentaux doit se conformer à la jurisprudence issue du droit international auquel adhère le Canada. Mentionnons à ce sujet que, depuis 1998, les rapports entre l'OIT et les pays membres de l'organisme ont changé. En raison des dispositions de la Déclaration de l'OIT relative aux principes et droits fondamentaux au travail et son suivi ${ }^{6}$, tous les pays membres de l'OIT (ce qui inclut le Canada) ont désormais l'obligation de respecter, de promouvoir et de réaliser, du seul fait de leur appartenance à l'Organisation et en l'absence de ratification des conventions pertinentes, les principes concernant les droits fondamentaux suivants: la liberté d'association et la reconnaissance effective du droit de négociation collective; l'élimination du travail forcé ou obligatoire, du travail des enfants, ainsi que de la discrimination en matière d'emploi et de profession (Duplessis, 2004: 53).

La place accordée au droit international en matière de liberté d'association en droit syndical semble évoluer de manière à éviter les distorsions entre la jurisprudence interne canadienne et la jurisprudence internationale (Bastarache, 2003: 374 et 378). Les juges de la Cour suprême du Canada semblent d'accord pour assurer une meilleure protection au droit à la négociation collective.

À quoi peut-on s'attendre lors des prochaines négociations collectives dans les secteurs public et parapublic? Celles-ci risquent-elles de se dérouler sans que le législateur adopte des lois spéciales qui ont pour effet d'interrompre la négociation ou (et) de suspendre l'exercice du droit de grève ${ }^{7}$ ?

La réponse à cette interrogation fondamentale en matière de liberté d'association en droit syndical ne saurait tarder. Les tribunaux auront, au cours des prochaines années, à trancher un différend entre les organisations syndicales et le gouvernement du Québec concernant la constitutionnalité de la Loi concernant les conditions de travail dans le secteur public (L.Q. 2005, chap. 43) (Perrier, 2007: 26-28), loi qui a eu

5. Au sujet de l'avenir de la négociation collective, le spécialiste en relations industrielles Gérard Hébert (1992: 1220) écrivait: «La négociation collective dans le secteur privé semble avoir devant elle un avenir prometteur. Le cadre où elle se déroule force les parties en présence à agir de façon raisonnable. Dans le cas du secteur public, tout invite les parties à agir de façon catégorique et péremptoire, mais cela conduit inévitablement à la négation même de la négociation. À moins que les agents de la négociation dans le secteur public ne se donnent un code d'éthique qu'ils respecteront malgré tout, malgré les inconvénients immédiats qui peuvent en découler, la négociation du secteur public est condamnée à disparaître.»

6. Déclaration de l'OIT relative aux principes et droits fondamentaux au travail, 1998, consulté sur Internet [http://www.ilo.org/ilolex/cgi-lex/pdconvf.pl ?host=status01\&textbase=ilofre \&document=2\&chapter=2 $6 \&$ query $=\% 28 \% 23$ docno $\% 3 \mathrm{D} 261998 \% 29+\% 40$ ref \&hightlight=\&querytype=bool\&context=0] le 10 janvier 2007.

7. De 1964 à 2006, l'Assemblée nationale du Québec a adopté (pour tous les secteurs d'activités économiques) 38 lois spéciales de retour au travail (Perrier, 2008: 46-48). 
pour effet d'imposer autoritairement, en décembre 2005, les conditions de travail et de rémunération aux salariés œuvrant dans les secteurs public et parapublic et qui a mis un terme aux grèves légales exercées par leurs organisations syndicales.

Toute intervention du Parlement ou des assemblées législatives dans le processus de négociation collective en droit du travail n'est pas complètement interdite. Aux yeux des juges de la Cour suprême du Canada, certaines situations peuvent justifier l'adoption de mesures d'exception compte tenu des circonstances particulières de chaque cas (RCS, 2004: 84-84). Dans la décision Terre-Neuve (Conseil du Trésor) c. N.A.P.E., les juges de la Cour suprême du Canada ont vérifié si «[l']article 9 de la Public Sector Act est justifiable au sens de l'article premier de la Charte». Ils ont examiné si «[1]a nécessité de remédier à la crise financière était un objectif législatif urgent et réel au printemps 1991 ». Au sujet de l'urgence d'agir pour le gouvernement de Terre-Neuve en 1991, les juges ont formulé les remarques suivantes:

La crise était grave. Les coûts nécessaires pour réaliser l'équité salariale selon l'échéancier initial représentaient une dépense importante. La baisse de la cote de crédit et son incidence sur la capacité d'emprunt du gouvernement ainsi que les coûts supplémentaires liés aux emprunts nécessaires pour financer la dette de la province étaient des questions très importantes. En outre, le gouvernement ne discutait pas seulement de droits par opposition à des dollars, mais également de droits par opposition à des lits d'hôpitaux, à des mises à pied, à des emplois, à l'éducation et à l'aide sociale.

Les tribunaux continueront de faire montre d'un grand scepticisme à l'égard des tentatives de justifier, par des restrictions budgétaires, des atteintes à des droits garantis par la Charte. Agir autrement aurait pour effet de déprécier la Charte étant donné qu'il y a toujours des restrictions budgétaires et que le gouvernement a toujours d'autres priorités urgentes. Cependant, les tribunaux ne peuvent pas fermer les yeux sur les crises financières périodiques qui, pour être surmontées, forcent le gouvernement à prendre des mesures pour gérer ses priorités. (RCS, 2004: 381)

Pour le moment, tout au plus pouvons-nous avancer que, pour les juges de la Cour suprême du Canada, ce que la Charte interdit concerne les interventions du législateur qui ont pour effet d'entraver de façon substantielle la négociation collective ou d'empêcher un syndicat d'exercer une véritable influence sur les conditions de travail de ses membres par l'entremise d'un processus de négociations mené de bonne foi (CSC, 2007: 89-90 et 92). L'avenir nous dira si la Loi concernant les conditions de travail dans le secteur public (L.Q. 2005, chap. 43) adoptée par le gouvernement Charest en décembre 2005 correspond en totalité ou non aux critères réputés inconstitutionnels aux yeux des juges de la Cour suprême.

\section{Conclusion}

L'interprétation que les juges de la Cour suprême du Canada font de la notion de liberté d'association a nettement évolué durant la période allant de 1982 à 2007. D'une vision restrictive, ayant pour effet de la réduire à sa plus simple expression de type formel et individuel, voilà que les juges de la Cour suprême du Canada ont décidé de reconnaître un aspect collectif et d'y inclure aussi le droit à la libre négociation en matière de droit syndical. En agissant de la sorte, les juges de la Cour suprême du Canada harmonisent la jurisprudence canadienne avec la jurisprudence internationale, ce qui va dans le sens initialement prévu par les rédacteurs de la 
Charte. À l'heure où l'on constate un déclin du syndicalisme dans notre société capitaliste marquée par la mondialisation, cette décision ne peut être que bénéfique pour la reconnaissance effective du droit de négociation pour les salariés syndiqués (Coutu, 2007: b5). De plus, ce revirement jurisprudentiel a pour effet de démontrer que le pouvoir judiciaire est en mesure de freiner, à l'occasion, le pouvoir politique quand celui-ci adopte des lois qui font fi des droits constitutionnels fondamentaux d'individus regroupés au sein de syndicats. Cette obligation juridique que les juges de la Cour suprême du Canada adressent aux élus de respecter des normes minimales élargies de justice sociale confirme la prééminence des tribunaux et vient, par ricochet, renforcer l'idée qu'on peut se faire de l'État de droit au Canada.

L'adoption de la Charte a fait passer le Canada «de la suprématie parlementaire à la suprématie constitutionnelle», donc de l'État légal à une forme d'État de droit. Nous disons «une forme d'État de droit», car cette suprématie constitutionnelle en regard des droits et libertés fondamentaux n'est pas absolue. Au Canada, le pouvoir législatif peut toujours recourir à divers moyens pour affirmer sa prééminence, et ce, même devant les décisions des tribunaux au sujet des droits et libertés fondamentaux.

Les négociations dans les secteurs public et parapublic au Québec ont donné lieu, jusqu'à maintenant, à des gestes qu'on peut apparenter à de l'autoritarisme étatique $^{8}$ et à un déni du droit à la négociation. Rappelons que la seizième ronde de négociations dans les secteurs public et parapublic s'est terminée, en décembre 2005, par l'adoption de la Loi concernant les conditions de travail dans le secteur public (L.Q. 2005, chap. 43). Cette loi a eu pour effets de mettre un terme à la grève des salariés et salariées des secteurs public et parapublic et d'imposer un décret d'une durée de six ans. Cette fin de négociation abrupte, marquée par l'adoption d'une loi spéciale de retour au travail accompagnée d'un décret, se voit contestée devant les tribunaux. À la suite des futures décisions des tribunaux, il nous sera possible de mieux cerner une notion centrale en droit et en science politique : la notion d'État de droit en regard de la portée et de l'étendue des libertés syndicales dans les secteurs public et parapublic. La liberté d'association, en matière de droit syndical, est-elle purement «légaliste, parcimonieuse et voire même insipide» (RCS, 1987: 81) ou est-elle incompatible avec toute expression d'autoritarisme étatique?

Il est arrivé à plus d'une reprise dans le passé que le gouvernement du Québec impose à ses salariés syndiqués des secteurs public et parapublic des solutions autoritaires. Pour faire triompher son point de vue, le gouvernement a, disons-le, souvent ignoré la loi. Il a fait adopter par le pouvoir législatif diverses lois d'exception ayant pour effet de suspendre l'exercice de certains droits (droit de négocier et droit de faire la grève). Il appartient maintenant à un acteur social réputé «neutre », l'acteur juridique, de se pencher sur les agissements du pouvoir politique. Il revient aux juges de dire si une loi qui a eu pour effet d'interrompre la négociation collective en décembre 2005 dans les secteurs public et parapublic au Québec est conforme ou non au contenu de la liberté d'association prévue à l'alinéa $2 \mathrm{~d}$ ) de la Charte. Cette loi spéciale adoptée par l'Assemblée nationale du Québec va à l'encontre probablement, du moins à sa face même, de la liberté d'association telle qu'interprétée aujourd'hui. Mais les droits constitutionnels ne sont pas absolus. Ils peuvent faire

8. «Ainsi l'État dans sa souveraineté aurait le choix de recourir selon les circonstances, l'inspiration de ses dirigeants, soit à des modes d'exercice démocratiques, soit à des modes d'exercice autoritaires.» (Abensour, 1997: X) 
l'objet de «limites raisonnables» et c'est souvent l'enjeu majeur des débats devant les tribunaux. Le rôle judiciaire de confirmer la «raisonnabilité» d'une mesure législative, expression de la volonté politique du jour, n'est pas nouveau; ce qui l'est, c'est qu'il porte maintenant sur des limitations aux droits fondamentaux. Cette loi correspond-elle à une manifestation d'autoritarisme étatique acceptable, au sein d'une société dite «libre et démocratique»(art. 1 de la Charte)?

Les décisions que les différents tribunaux auront à prendre au sujet des contestations juridiques de la Loi concernant les conditions de travail dans le secteur public (L.Q.2005, chap. 43) nous permettront de dégager l'étendue et la portée concrète des droits que les salariés syndiqués des secteurs public et parapublic peuvent opposer à un gouvernement tenté de régler un différend à l'extérieur des règles prévues par la loi du régime de négociation. Il nous sera dès lors possible d'intégrer, dans notre analyse théorique de l'État de droit, ce qui est réputé juridiquement fondé selon les tribunaux. La nouvelle théorie qui en résultera pourra nous renseigner un peu plus adéquatement sur le potentiel réel d'action des pouvoirs exécutif et législatif dans des situations dites d'urgence. Jusqu'à quel point ces pouvoirs peuvent-ils, en toutes circonstances, se soustraire à leurs obligations inscrites dans la loi et dans les traités internationaux, pour imposer leurs points de vue lors des négociations dans les secteurs public et parapublic?

Les tribunaux ont à nous éclairer sur la signification réelle de l'État de droit en matière de régime de liberté syndicale. Ou bien le gouvernement est lié par les lois que l'Assemblée nationale a adoptées, ou bien il peut les contourner impunément quand bon lui semble. Autrement dit, ou bien les salariés syndiqués des secteurs public et parapublic peuvent jouir pleinement des droits inhérents à la liberté d'association syndicale ou bien les droits associés à cette liberté correspondent à un régime de «droits fictifs», bref à un régime de négociation «de façade».

Il revient donc au droit d'alimenter la réflexion de la science politique au sujet de cet enjeu important concernant une liberté fondamentale pour plus de 500000 salariés syndiqués œuvrant dans les secteurs public et parapublic au Québec.

\section{Liste des organisations syndicales mentionnées dans le texte}

CEQ: Centrale de l'enseignement du Québec

CSN: Confédération des syndicats nationaux

CSQ: Centrale des syndicats du Québec

FEESP: Fédération des employées et employés des services publics

FIIQ: Fédération des infirmières et infirmiers du Québec

FP: Fédération des professionnèles

FSSS: Fédération de la santé et des services sociaux

FTQ: Fédération des travailleurs et travailleuses du Québec

SFPQ: Syndicat des fonctionnaires de la province de Québec 


\section{Bibliographie}

Abensour, Miguel, 1997, La démocratie contre l'État. Marx et le moment machiavélien, Paris, Presses universitaires de France.

Arthurs, Harry W., 2007, «Labour and the "Real" Constitution», Les Cahiers de Droit, vol. 48, $\mathrm{n}^{\circ} 3$.

Bastarache, Michel, 2003, «The Canadian Charter of Rights and Freedom: Domestic Application of Universal Values», Supreme Court Law Review, 19.

Bendel, Michael, 1986, «La liberté d'association dans l'optique de la Charte canadienne des droits et libertés», dans Daniel Turp et Gérald A. Beaudoin (sous la dir. de), Perspectives canadiennes et européennes des droits de la personne, Cowansville, Les Éditions Yvon Blais Inc., p. 322-349.

Brunelle, Christian et Pierre Verge, 2003, «L'inclusion de la liberté syndicale dans la liberté générale d'association: un pari constitutionnel perdu? », Revие du barreau canadien, $\mathrm{n}^{\circ} 82$.

Brunelle, Christian, Michel Coutu et Gilles Trudeau, 2007, «La constitutionnalisation du droit du travail: un nouveau paradigme», Les Cahiers de Droit, vol. 48.

Caire, Guy, 1976, Liberté syndicale et développement économique, Genève, Bureau international du travail.

Carré de Malberg, Raymond, 2004 [1920], «La distinction de l'État légal et de l'État de droit», Problèmes politiques et sociaux, $\mathrm{n}^{\circ}$ 898, p. 23-25.

CEQ et CSN, 1984, Histoire du mouvement ouvrier au Québec: 150 ans de luttes, Montréal, Coédition CEQ-CSN.

Cour supérieure du Québec, 2007, Sous la présidence de l'Honorable Claudine Roy, J.C.S.

Cour suprême du Canada, 1987, Recueil des arrêts de la Cour Suprême du Canada, Renvoi relatif à la Public Service Employee Relations Act (Alb.), 1 RCS.

Cour suprême du Canada, 1990a, Recueil des arrêts de la Cour Suprême du Canada, Mahe $c$. Alberta, 1 RCS.

Cour suprême du Canada, 1990b, Recueil des arrêts de la Cour Suprême du Canada, Institut professionnel de la fonction publique du Canada c. Territoires du Nord-Ouest (Commissaire), 2 RCS.

Cour suprême du Canada, 1997, Recueil des arrêts de la Cour Suprême du Canada, Godbout c. Ville de Longueuil, 3 RCS.

Cour suprême du Canada, 1998, Recueil des arrêts de la Cour Suprême du Canada, Renvoi relatif à la sécession du Québec, 2 RCS.

Cour suprême du Canada, 1999a, Recueil des arrêts de la Cour Suprême du Canada, Baker c. Canada (Ministre de la Citoyenneté et de l'Immigration), 2 RCS.

Cour suprême du Canada, 1999b, Recueil des arrêts de la Cour Suprême du Canada, Delisle c. Canada (Sous-procureur général), 2 RCS.

Cour suprême du Canada, 2001, Recueil des arrêts de la Cour Suprême du Canada, Dunmore c. Ontario (Procureur général), 3 RCS.

Cour suprême du Canada, 2004, Recueil des arrêts de la Cour Suprême du Canada, Terre-neuve (Conseil du Trésor) c. N.A.P.E., 3 RCS.

Cour suprême du Canada, 2005, Recueil des arrêts de la Cour Suprême du Canada, Chaouili c. Québec (Procureur général), 1 RCS.

Cour suprême du Canada, 2007, Recueil des arrêts de la Cour Suprême du Canada, Health Services and Support - Facilities Subsector Bargaining Assn, c. Colombie-Britannique, CSC 27. 
Coutu, Michel, 2007, «L'arrêt Health Services and Support de la Cour suprême du Canada: La constitutionnalisation du régime québécois des relations industrielles? », Le Devoir, Montréal, 29 juillet, p. b5.

Coutu, Michel et Georges Marceau, 2007, Droit administratif du travail: Tribunaux et organismes spécialisés du domaine du travail, Montréal/Cowansville, CRIMT/Éditions Yvon Blais.

Duplessis, Isabelle, 2004, «La déclaration de l'OIT relative aux droits fondamentaux au travail: Une nouvelle forme de régulation efficace? », Relations industrielles, vol. 59, n 1, p. 52-72.

Habermas, Jürgen, 1992, Droit et démocratie. Entre faits et normes, coll. «NRF essais», Paris, Gallimard.

Hébert, Gérard, 1992, Traité de négociation collective, Boucherville, Gaëtan Morin éditeur.

Lévesque, Lia, 2007, «Secteur public: Les syndicats veulent bien revoir le régime de négociations », Le Devoir, Montréal, vendredi 15 juin, p. A3.

Notebaert, Gérard, 2007, Les rapports collectifs de travail chez les agents de la paix en services correctionnels et les policiers de la Sûreté du Québec: une contravention à la liberté d'association, texte photocopié, Montréal, Université du Québec à Montréal.

Organisation internationale du travail (OIT), 1998, Déclaration de l'OIT relative aux principes et droits fondamentaux au travail, consulté sur Internet [http://www.ilo.org/ilolex/ cgi-lex/pdconvf.pl ?host $=$ status01\& textbase $=$ ilofre $\&$ document $=2 \&$ chapter $=26 \& q$ uery $=\% 28 \% 23$ docno $\% 3 \mathrm{D} 261998 \% 29+\%$ 40ref \& hightlight $=\&$ querytype=bool $\&$ con text $=0$ ] le 10 janvier 2007.

Panitch, Leo et Donald Swartz, 1988, The Assault on Trade Union Freedoms: From Consent to Coercition Revisited, Toronto, Garamond Press.

Perrier, Yvan, 1992, Étude de certaines théories de la régulation et analyse de la régulation étatique des rapports collectifs de travail dans les secteurs public et parapublic au Québec de 1964 à 1986 (de la libre contractualisation à la négociation factice), thèse de doctorat, Montréal, Université du Québec à Montréal.

Perrier, Yvan, 1999, Les négociations dans les secteurs public et parapublic: effectivité du droit, Montréal, Centre de recherche en droit public.

Perrier, Yvan, 2000, De l'État de droit à l'État démo-autoritaire. Une analyse de cas: les négociations dans les secteurs public et parapublic au Québec de 1964 à 1999, XVIII ${ }^{\mathrm{e}}$ Congrès mondial de l'Association internationale de science politique, Québec.

Perrier, Yvan, 2007, Négociations dans les secteurs public et parapublic au Québec de 2002 à 2006: retour critique sur un décret annoncé sur le tard, Colloque 2007 de la Société québécoise de science politique, Québec.

Perrier, Yvan, 2008, Constitutionnalisation de la liberté d'association au Canada et négociation des rapports collectifs de travail dans les secteurs public et parapublic au Québec, Colloque 2008 de la Société québécoise de science politique, Montréal.

Richer, Jocelyne, 2008, «Négociations avec le secteur public; Québec veut revoir les règles du jeu», Le Devoir, Montréal, 26 août, p. b1.

Rouillard, Jacques, 2004, Le syndicalisme québécois: Deux siècles d'histoire, Montréal, Boréal.

Tremblay, Guy, 2005, «Le pouvoir judiciaire», dans Réjean Pelletier et Manon Tremblay (sous la dir. de), Le parlementarisme canadien, Québec, Presses de l'Université Laval, p. 411-438.

Trudeau, Gilles, 2001, «Droit international et droit du travail québécois, deux grandes solitudes», dans Service de la formation permanente du barreau du Québec, Développement récents en droit du travail, Cowansville, Les Éditions Yvon Blais inc.

Woehrling, José, 2005, «La Charte canadienne des droits et libertés et ses répercussions sur la vie politique», dans Réjean Pelletier et Manon Tremblay (sous la dir. de), Le parlementarisme canadien, Québec, Presses de l'Université Laval, p. 81-118. 